\title{
Effects of increased compression with an ultrasound transducer on the conspicuity of breast lesions in a phantom
}

Katy Szczepura, Tahreem Faqir, David Manning

Katy Szczepura, Tahreem Faqir, David Manning, "Effects of increased compression with an ultrasound transducer on the conspicuity of breast lesions in a phantom," Proc. SPIE 10136, Medical Imaging 2017: Image Perception, Observer Performance, and Technology Assessment, 101360T (24 April 2017); doi: 10.1117/12.2254263

SPIE. Event: SPIE Medical Imaging, 2017, Orlando, Florida, United States 


\title{
Effects of Increased Compression with an Ultrasound Transducer on the
}

\section{Conspicuity of Breast Lesions in a Phantom}

\author{
Katy Szczepura, Tahreem Faqir, David Manning \\ University of Salford, Manchest er, UK
}

\begin{abstract}
Ultrasound imaging of the breast is highly operator dependant. The amount of pressure applied with the transducer has a direct impact on the lesion visibility in breast ultrasound.

The conspicuity index is a quantitative measure of lesion visibility, taking into account more parameters than standard measures that impact on lesion detection [1].

This study assessed the conspicuity of lesions within a breast phantom using increased transducer compression in breast ultrasound.

\section{Methods}

A phantom was constructed of gelatine to represent adipose tissue, steel wool for glandular/blood vessels and silicone spheres to represent lesions, this meant that the lesions were also compressible, but less than the surrounding tissue.
\end{abstract}

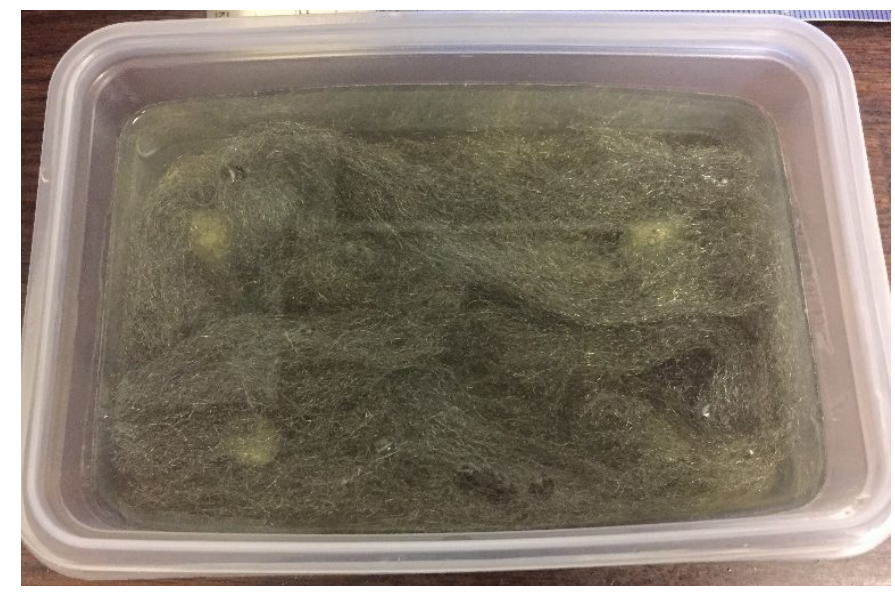

The phantom was imaged under increasing transducer compression. 


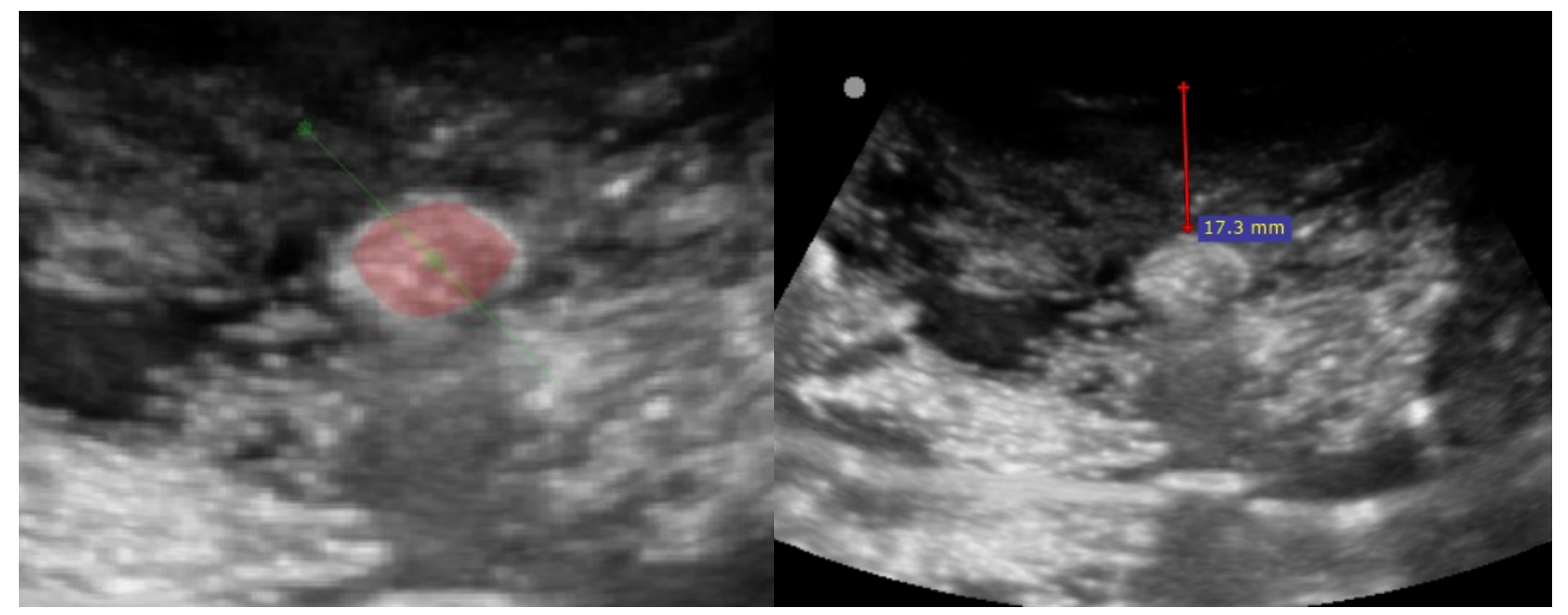

The conspicuity index was measured using the Conspicuity Index Software. The distance between the transducer surface and lesion surface was measured as an indication of increased compression.

\section{Results}

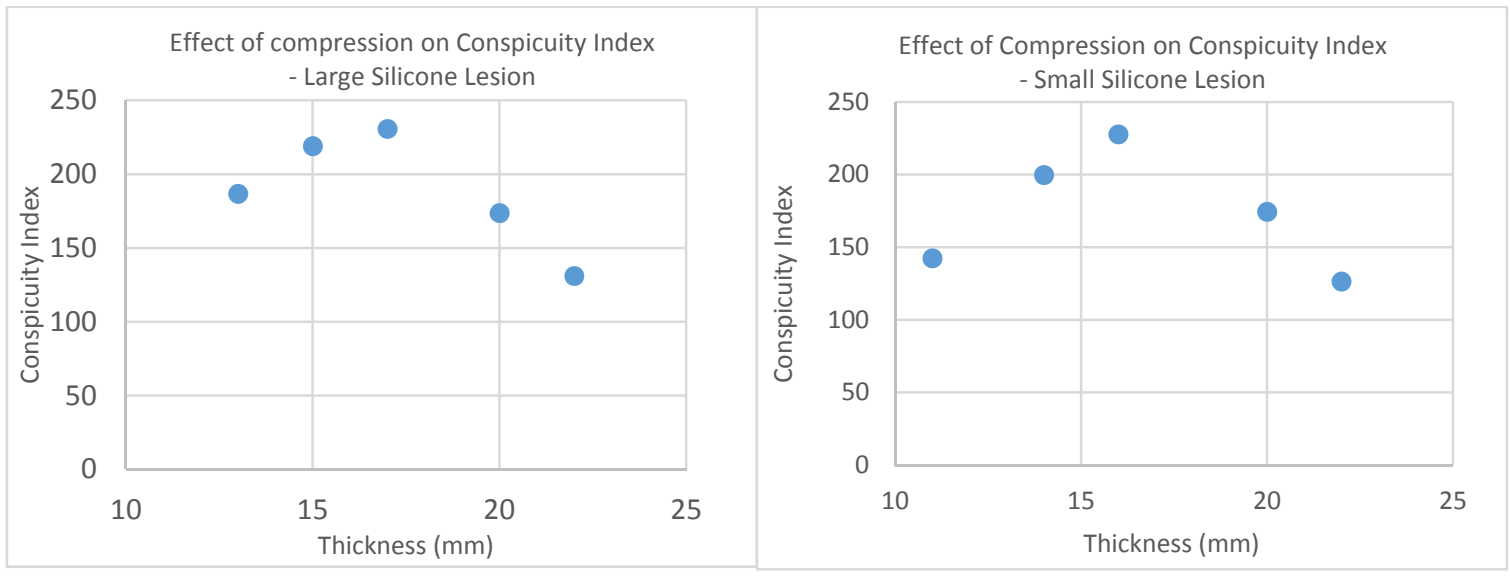

When moderate compression $(17 \mathrm{~mm})$ was applied, the conspicuity index increased resulting in better visualisation of the silicone lesions. However, with increased compression the conspicuity index decreased.

\section{New work to be presented}

The conspicuity index has never been demonstrated in ultrasound imaging before. This is preliminary phantom work to demonstrate the impact of increased transducer compression on quantitative lesion visibility assessment.

\section{Conclusion}

The compression applied should be moderate for optimum visualisation, as excessive pressure decreases conspicuity. However, further work needs to be conducted in order to consider other factors, such as density of the breast and lesion location, for a better understanding of the effect of compression on the visualisation of the lesion. A human study is planned. 


\section{INTRODUCTION}

Ultrasound scanning is often used in conjunction with mammograms and are performed when additional information is required. Ultrasound is used to explore factors, such as the source of breast symptoms, check for breast lumps and investigate abnormal results from a mammogram. In addition, it is frequently a choice of imaging for young women with dense breast tissue, as mammograms do not demonstrate sufficient detail [2] Although, ultrasound is used in addition to a mammogram, it can be the primary choice for detecting breast lesions. This often occurs when a mammogram is not an option, due to the ionising radiation, or simply is not available [2]. Ultrasound has many characteristics, which make it an extremely useful modality. These characteristics include: simplicity (it does not require any special preparation), immediacy of the image, rapidity of examination, availability, ability to study pathologic and normal masses in real time, cost and that it does not carry any known side effects. Ultrasound is usually not uncomfortable, however discomfort can be experienced when the transducer is compressed against the area of examination [3].

Ultrasound is able to detect palpable, as well as non-palpable lesions and early carcinomas. In addition, it is often used for the staging of the lesions. However, the accuracy of the ultrasound examinations is highly dependent on the examiner's experience and the equipment [4]. Ultrasound is commonly stated as being "operator dependent" in that the examination is dependent on the dedication and experience of the operator. A suboptimal ultrasound scan can be interpreted in many different ways, which makes ultrasound a less objective technique in comparison to mammograms [3].

Reflecting structures at severe angles to the beam of the ultrasound can cause excessive shadow artefacts and reduce image quality due to the reduction of penetration [5]. During the scanning of a breast lesion, angulation and compression of the transducer is a common technique to sharpen the edges of the lesion [6]. The degree of compression applied can vary and is often dependent on the location of the lesion, the pendulousness and size of the breast.

The compression applied in combination with the patient's positioning are key elements in the ultrasound of the breast and play a vital role in discovering and the visualisation of a lesion. The patient is scanned in a contralateral posterior oblique or a supine position and they are asked to place their arms behind their head [2], this thins the tissue being scanned and forces the normal breast tissue into a parallel plane in relation to the transducer and perpendicular to the beam. This improves the conspicuity of lesions and the overall image quality by allowing greater beam penetration, as compression can decrease refraction and scatter from neighbouring structures [7]. 
Increased pressure can be applied to lesions that are small, have fibrous tissue lying superficially and are situated deeply near the chest wall. Occasionally, structures that lie superficial to the lesion, e.g. Cooper's ligament, can create acoustic shadows and hinder the evaluation of deeper structures. With the use of moderate compression this can be eliminated [7]. However, extensive compression with the use of a transducer may not always improve image quality. Vigorous compression can result in the attenuation or even the elimination of vascular signals when using Doppler, predominantly in benign lesions [3]. Furthermore, scanning in a perpendicular plane to the near-field tissue in combination with heavy compression, has the ability to push side-lobe artefacts and near-field reverberation echoes deeper into the breast. This can interfere with the evaluation of superficial lesions and consequently have a detrimental effect on the diagnosis and patient's pathway. In such cases, the visualisation can be improved by not applying any compression or with the use of angulation. This allows the beam to penetrate obliquely through the lesion, rather than perpendicularly [7].

This is supported by Carson et al, who proposes that deeper structures have reduced acoustic path lengths when considerable local compression is applied [5]. It is demonstrated by Fargier-Voiron et al, that excessive pressure, applied by the transducer, has the ability to impact visualisation and localisation of lesions. They further recommend, to achieve better accuracy, the transducer pressure should be kept as low as possible. This, in addition, will allow sufficient contact for optimum image quality [8].

Furthermore, it has been proposed by Dobler et al, a probe displacement of 1-2 cm was essential in order to obtain good image quality. The study established that excessive compression can result in a shift of anatomical structures [9] consequently, making it difficult to localise lesions. This study, in combination with multiple other studies, demonstrated a maximum displacement of approximately $1.7 \mathrm{~cm}$ when excessive pressure was applied [10-12] Although, these studies were conducted on the displacement of prostates, the same principle can be applied to the ultrasound scanning of breasts.

\section{METHODOLOGY}

\section{Phantom Design}

In order to investigate new imaging techniques, it is vital that the phantom used has the properties to mimic human tissue. Although, commercial ones are an easy option, they are not very easily adaptable to specific applications. Inhouse, custom made options allow an individual to tailor their phantom to their needs. There are several studies that promote the use of gelatine to create a phantom [13]. According to Farrer et al, gelatine has properties in the same order of magnitude as that of soft tissue and is easy to manufacture [14]. Therefore, in order to simulate breasts, a gelatine phantom was made. A study conducted by Sultan et al suggested using $70 \mathrm{~g}$ (6 sachets) of Dr Oetker's gelatine and $500 \mathrm{ml}$ of water, which resulted in the gelatine-to-water ratio of approximately 1:7 [15]. However, when this ratio was used, the 
density was too firm and did not resemble human tissue. After some trial-and-error, the gelatine-to-water ratio used was $1: 31$, using $18 \mathrm{~g}$ (1.5 sachets) of Dr Oetker's gelatine and $570 \mathrm{ml}$ of water.

The $570 \mathrm{ml}$ of hot water was poured into a jug and the $18 \mathrm{~g}$ of gelatine was sprinkled into the water very slowly, whilst the mixture was being stirred, allowing the gelatine to dissolve completely.

The lesions were made from, silicone spheres, two lesions, measuring approximately $1 \mathrm{~cm}$ and $1.5 \mathrm{~cm}$, were made. In order to simulate glandular tissue, fine steel wool was used [16]. The steel wool was placed in a small plastic tub, which measured $15 \mathrm{~cm} \times 10 \mathrm{~cm} \times 7 \mathrm{~cm}$. The lesions were placed within the steel wool towards the opposite corners of the tub and the mixture of water and gelatine was poured into the plastic tub. It was essential to ensure the mixture covered the entire steel wool to prevent the formation of air bubbles (Figure 1).

Figure 1 phantom construction

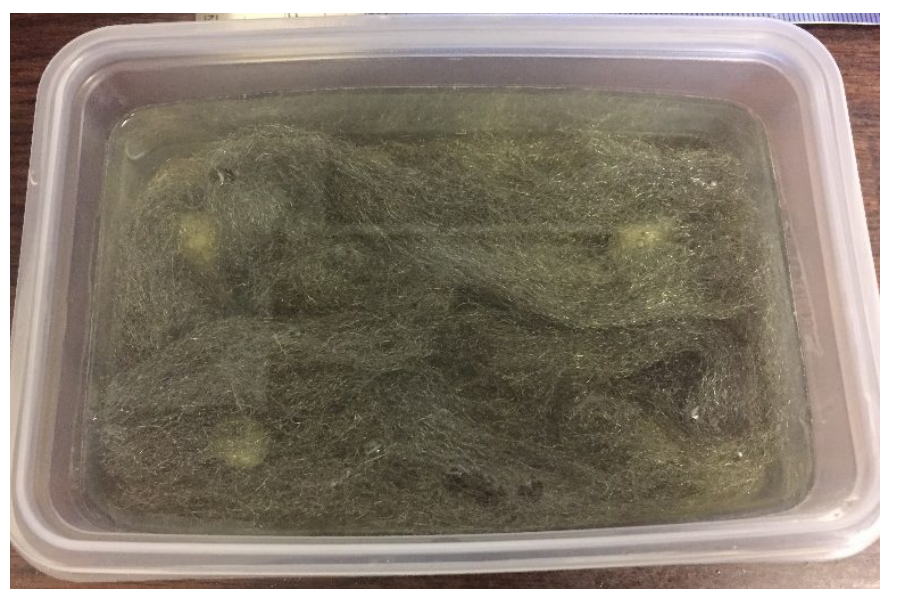

The tub was then refrigerated until 2 hours before it was required for scanning. The total depth of the phantom was $4.5 \mathrm{~cm}$.

\section{Image Acquisition}

The images were acquired using a SonoSite M-Turbo Ultrasound scanner with a C60X transducer. The transducer was used to apply pressure to the phantom with the aid of "Aquasonic 100 Ultrasound Gel”. Each of the lesions was scanned with 5 different compressions. The pressure applied varied from minimal compression (compression 1), just enough to obtain sufficient image quality, to extreme compression (compression 5). The aim throughout was to maintain equal changes in pressure. During each compression, the screen was frozen and the image was exported as DICOM images. Variables, such as windowing or the field-of-view were kept constant, this was to ensure that the change of visualisation was only caused by the change in compression. 


\section{Conspicuity index analysis}

Conspicuity software (ref) was used to draw a region of interest (ROI) around the abnormality (

Figure 2) avoiding artefacts.

Figure 2 ROI within the conspicuity index software

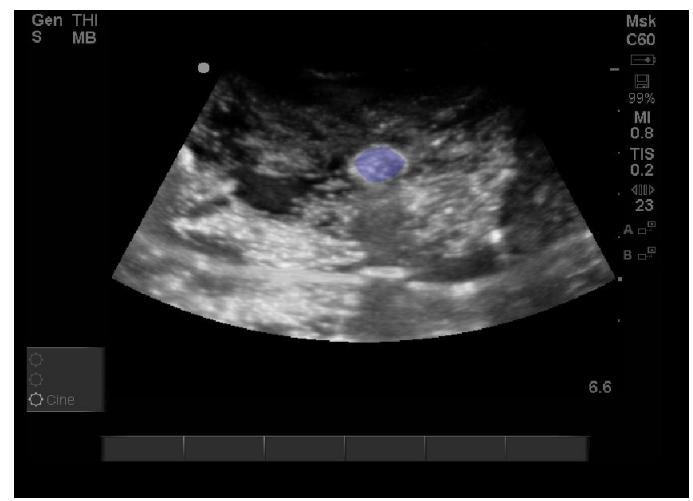

The programme then plotted 180 line profiles around the region drawn, which resulted in 360 edge profiles.

A fit was then applied for every line profile (Figure 3). The blue line demonstrated the line profile, the red line represented the plotted fit and the green line demonstrated the ROI drawn by the operator. This programme then acquired the pixel values of the lesion and the background. It calculated the standard deviation for these values, which represented the lesion and anatomical noise.

Figure 3 Line profile

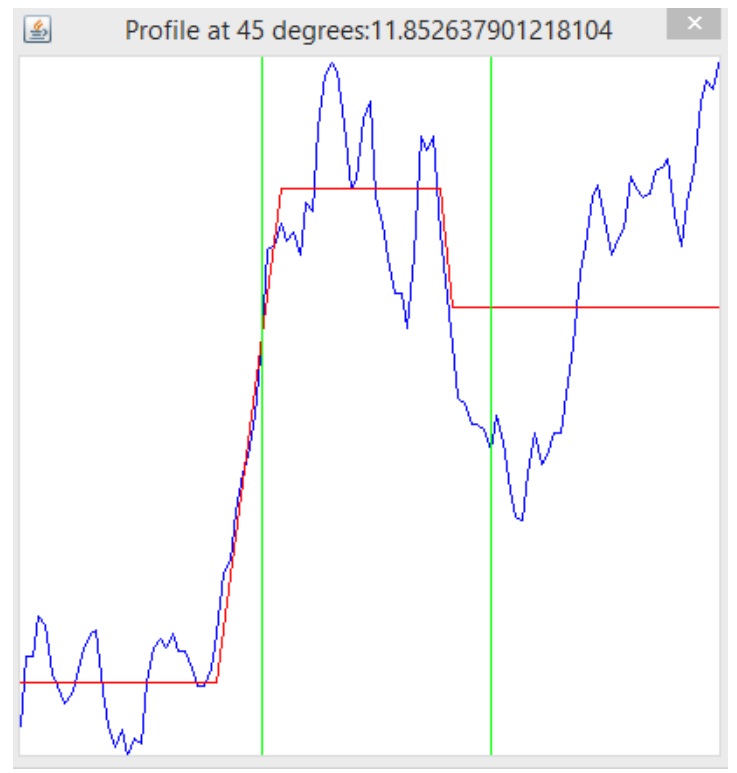


Figure 4 Example single line profile across the region of interest for a lesion

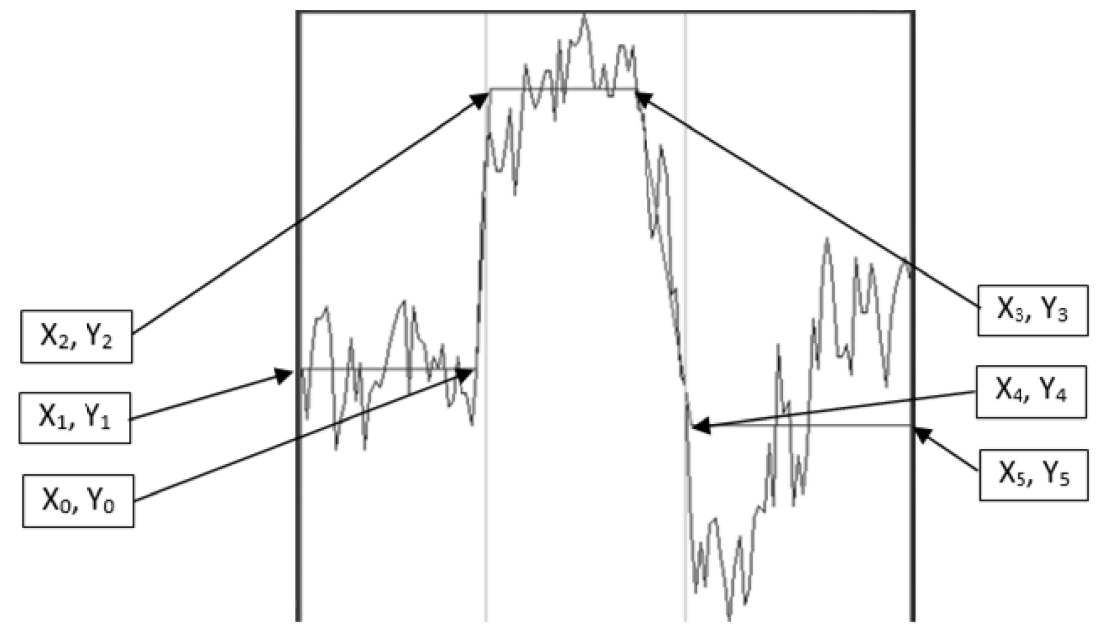

An Excel spreadsheet has been developed to import the TSV files and then to calculate the following:

$$
\begin{gathered}
\bar{d}=\overline{(X 3-X 2)}=\text { average lesion dimension } \\
\text { Equation } 1
\end{gathered}
$$

$$
\bar{\theta}=\overline{\tan ^{-1}\left[\frac{Y_{2}-Y_{1}}{X_{2}-X_{1}}\right], \tan ^{-1}\left[\frac{Y_{4}-Y_{3}}{X_{4}-X_{3}}\right]}=\text { average slope angle of all the line profiles }
$$

Equation 2

$$
\begin{gathered}
\overline{\Delta G L}=\overline{Y_{2}-\frac{\left(Y_{1}+Y_{4}\right)}{2}=\text { the average difference in grey levels between the lesion and the background }} \\
\text { Equation } 3 \\
\overline{\sigma_{s}}=\text { sdTop }
\end{gathered}
$$

Equation 4

$$
\overline{\sigma_{n}}=\frac{s d 1+s d 2}{2}
$$

\section{Equation 5}

This data was then used to calculate the Conspicuity Index $(\chi)$ :

$$
\chi=\frac{\bar{d} \tan [\bar{\theta}-1] \overline{\Delta G L}}{\sqrt{\overline{\sigma_{s}^{2}}+\overline{\sigma_{n}^{2}}}}
$$


The ROI was drawn around each lesion three times, to work out the standard deviation and measure reproducibility. From the three conspicuity index numbers, an average was calculated.

After this data was collected, the original ultrasound images were used to calculate the pixels and $\mathrm{mm}$ of the top of the ultrasound image to the top of the lesion (Figure 5). This was used to work out the change in thickness above the lesion in $\mathrm{mm}$ to indicate the level of compression.

Figure 5 measuring thickness from transducer to surface of lesion

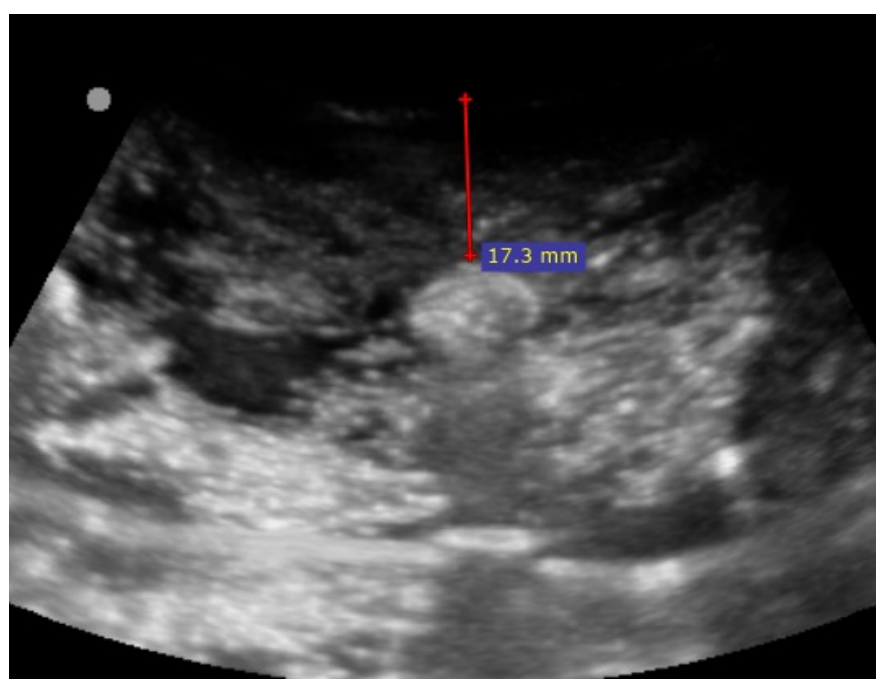




\section{RESULTS}

Graph 1 Effect of compression on C.I. for $1.5 \mathrm{~cm}$ lesion

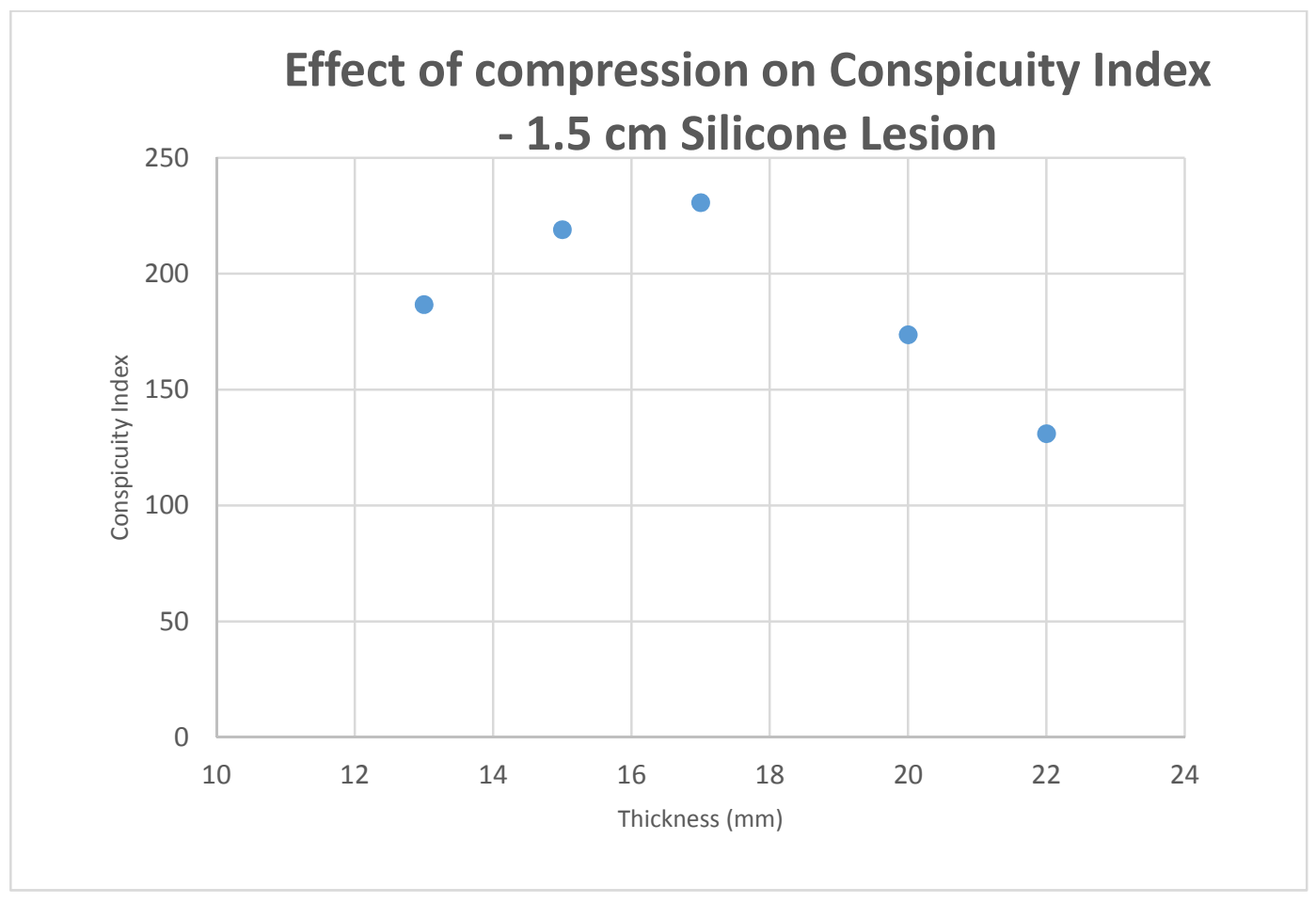

Graph 2 Effect of compression on C.I. for $1 \mathrm{~cm}$ lesion

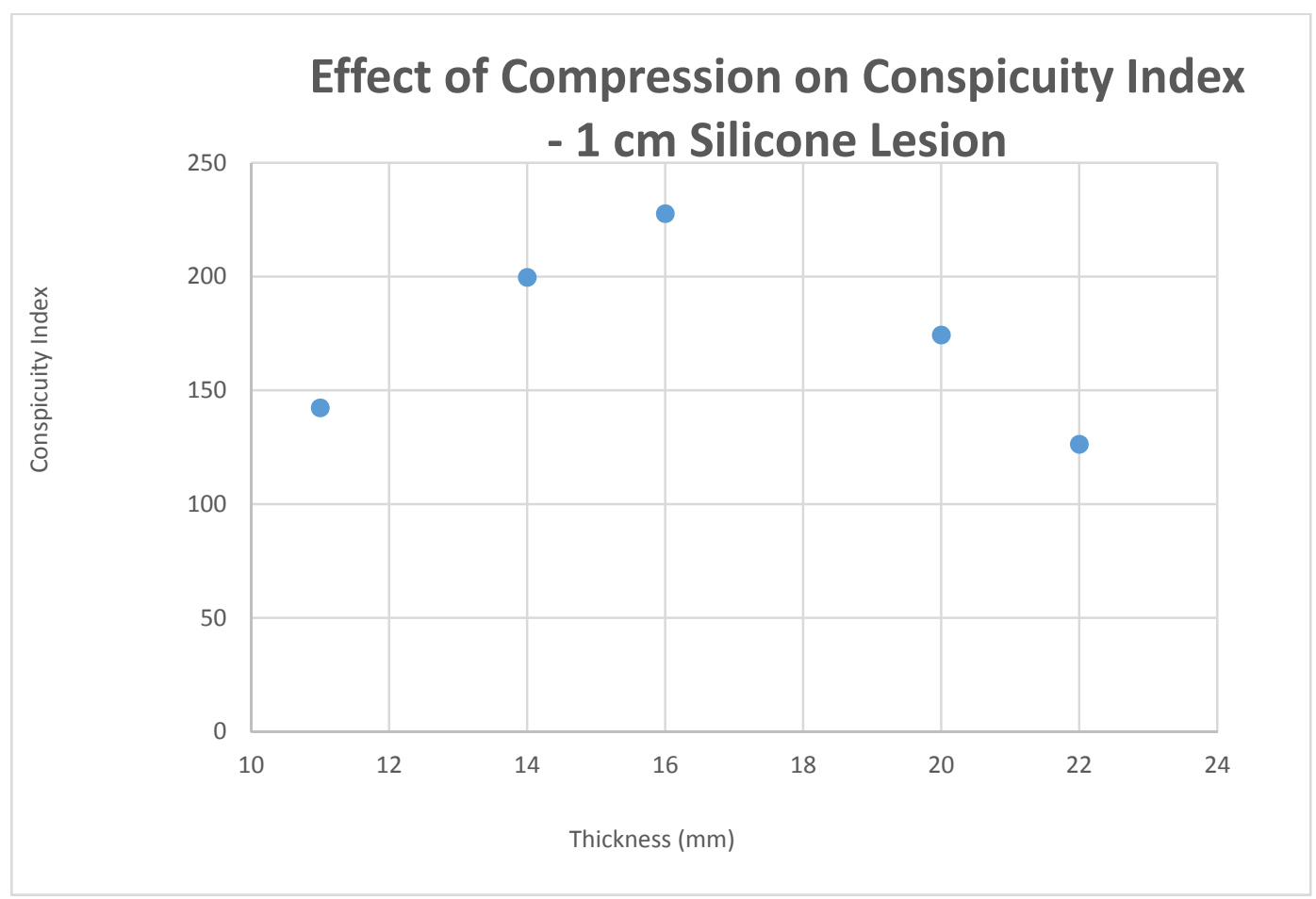

Proc. of SPIE Vol. 10136 101360T-9 


\section{DISCUSSION}

Overall, the results show when compression is applied, the conspicuity index of the lesion can improve. The compression applied should be between minimal and moderate for optimum visualisation, as excessive pressure decreases the conspicuity index. Graph 1Graph 2 establishes that the optimum compression for the silicone lesions was at a thickness reduction of $17 \mathrm{~mm}$, when applying moderate compression, regardless of size. The conspicuity index was at its highest at approximately 230 for both the lesions.

It is important to recognise that in addition to compression, the image quality and the visualisation of a lesion is impacted by multiple other factors in ultrasound imaging. These elements include the patient's breast size and density, the equipment used, and the amount and distribution of gel [17].

\section{CONCLUSION}

The compression applied should vary from minimal to moderate, as excessive pressure can result in loss of conspicuity and hinder detectability. Consequently, this can result in false negatives and impact the patient's diagnosis and pathway. However, further research is required to take other factors that can affect the visualisation into consideration.

A patient study is planned to see if this preliminary work can be replicated in humans.

\section{REFERNCES}

[1] K. R. Szczepura, and D. J. Manning, "Validated novel software to measure the conspicuity index of lesions in DICOM images," 9787, 978703-978703-15 (2016).

[2] A.-M. M. Dixon, [Breast ultrasound : how, why and when] Churchill Livingstone Elsevier, Edinburgh(2008).

[3] O. Catalano, A. Nunziata, and A. Siani, [Fundamentals in Oncologic Ultrasound: Sonographic Imaging and Intervention in the Cancer Patient] Springer Science \& Business Media, (2009).

[4] H. Madjar, H. A. Ladner, W. Sauerbrei et al., "Preoperative staging of breast cancer by palpation, mammography and high-resolution ultrasound," Ultrasound in Obstetrics \& Gynecology: The Official Journal of the International Society of Ultrasound in Obstetrics and Gynecology, 3(3), 185-190 (1993).

[5] P. L. Carson, B. Wang, G. L. LeCarpentier et al., "Local compression in automated breast ultrasound in the mammographic geometry." 1787-1790.

[6] N. Yang, "Breast ultrasound | Radiology Reference Article | Radiopaedia.org."

[7] A. T. Stavros, [Breast Ultrasound] Lippincott Williams \& Wilkins, (2004).

[8] M. Fargier-Voiron, B. Presles, P. Pommier et al., "Impact of probe pressure variability on prostate localization for ultrasound-based image-guided radiotherapy," Radiother Oncol, 111(1), 132-7 (2014).

[9] B. Dobler, S. Mai, C. Ross et al., "Evaluation of possible prostate displacement induced by pressure applied during transabdominal ultrasound image acquisition," Strahlentherapie Und Onkologie: Organ Der Deutschen Rontgengesellschaft ... [et Al], 182(4), 240-246 (2006). 
[11] J. P. McGahan, J. Ryu, and M. Fogata, "Ultrasound probe pressure as a source of error in prostate localization for external beam radiotherapy," International Journal of Radiation Oncology, Biology, Physics, 60(3), 788-793 (2004).

[12] C. F. Serago, S. J. Chungbin, S. J. Buskirk et al., "Initial experience with ultrasound localization for positioning prostate cancer patients for external beam radiotherapy," International Journal of Radiation Oncology, Biology, Physics, 53(5), 1130-1138 (2002).

[13] J. Sutcliffe, R. L. Hardman, N. C. Dornbluth et al., "A novel technique for teaching challenging ultrasound-guided breast procedures to radiology residents," Journal of Ultrasound in Medicine: Official Journal of the American Institute of Ultrasound in Medicine, 32(10), 1845-1854 (2013).

[14] A. I. Farrer, H. Odéen, J. de Bever et al., "Characterization and evaluation of tissue-mimicking gelatin phantoms for use with MRgFUS," Journal of Therapeutic Ultrasound, 3, (2015).

[15] S. F. Sultan, G. lohom, and G. Shorten, "A Novel Phantom for Teaching and Learning Ultrasoundguided Needle Manipulation," Journal of Medical Ultrasound, 21(3), 152-155 (2013).

[16] Y. Li, “Mammographic Density Assessment: Inter-Reader Variability And Novel Phantom Quantification," (2015).

[17] P. Chatelain, A. Krupa, and N. Navab, "Optimization of ultrasound image quality via visual servoing." 5997-6002. 